PROCEEDINGS OF THE

AMERICAN MATHEMATICAL SOCIETY

Volume 133, Number 10, Pages 2957-2964

S 0002-9939(05)07777-4

Article electronically published on May 13, 2005

\title{
THE ROKHLIN LEMMA FOR HOMEOMORPHISMS OF A CANTOR SET
}

\author{
S. BEZUGLYI, A. H. DOOLEY, AND K. MEDYNETS
}

(Communicated by Michael Handel)

\begin{abstract}
For a Cantor set $X$, let $\mathrm{Homeo}(X)$ denote the group of all homeomorphisms of $X$. The main result of this note is the following theorem. Let $T \in \operatorname{Homeo}(X)$ be an aperiodic homeomorphism, let $\mu_{1}, \mu_{2}, \ldots, \mu_{k}$ be Borel probability measures on $X$, and let $\varepsilon>0$ and $n \geq 2$. Then there exists a clopen set $E \subset X$ such that the sets $E, T E, \ldots, T^{n-1} E$ are disjoint and $\mu_{i}\left(E \cup T E \cup \ldots \cup T^{n-1} E\right)>1-\varepsilon, i=1, \ldots, k$. Several corollaries of this result are given. In particular, it is proved that for any aperiodic $T \in \operatorname{Homeo}(X)$ the set of all homeomorphisms conjugate to $T$ is dense in the set of aperiodic homeomorphisms.
\end{abstract}

\section{INTRODUCTION}

One of the most useful results in ergodic theory which has many important applications is the Rokhlin lemma $[\underline{R}$. This statement asserts that given an aperiodic (non-singular) automorphism $T$ of a standard measure space $(X, \mathcal{B}, \mu), \varepsilon>0$, and $n \geq 2$, there exists a measurable subset $E \subset X$ such that $E, T E, \ldots, T^{n-1} E$ are pairwise disjoint and $\mu\left(E \cup T E \cup \ldots \cup T^{n-1} E\right)>1-\varepsilon$. It immediately follows from this result that the set of periodic automorphisms is dense in the group of all nonsingular automorphisms of $(X, \mathcal{B}, \mu)$ with respect to the metric $d\left(T_{1}, T_{2}\right)=\mu\left\{\left(x \in X: T_{1} x \neq T_{2} x\right)\right\}$ where $T_{1}, T_{2} \in \operatorname{Aut}(X, \mathcal{B}, \mu)$. Subsequently, the Rokhlin lemma was generalized in various directions (see, for example, AP $, \mathrm{EP}, \mathrm{FL}, \mathrm{LW}, \mathrm{OW}]$ ). It is well known that the Rokhlin lemma is also related to amenability.

Our goal is to prove a version of the Rokhlin lemma in the context of Cantor dynamics. To start with, we need to consider a topology on $\mathrm{Homeo}(X)$ analogous to the metric $d$. It is well known that $\operatorname{Homeo}(X)$ equipped with the topology $\tau_{w}$ of uniform convergence (the sup-topology) is a Polish space. But $\tau_{w}$ is too weak to trace the dynamical properties of homeomorphisms. For instance, the set of

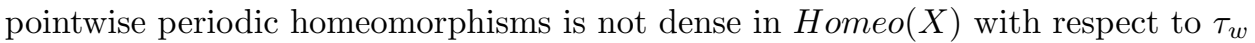
(for more details see BDK1, BDK2]). In [BK1, BK2, BDK1, BDK2], we introduced and studied a topology $\tau$ which has its origin in measurable dynamics. It was shown in BDK1, BDK2 that $\tau$ plays the same role in Borel and Cantor dynamics as the metric $d$ plays in ergodic theory. Recall the definition of the uniform topology $\tau$. For

Received by the editors October 20, 2003.

2000 Mathematics Subject Classification. Primary 37H15, 37B05; Secondary 54H20.

(C)2005 American Mathematical Society 
$T, S \in \operatorname{Homeo}(X)$, define $E(S, T)=\{x \in X: T x \neq S x\} \cup\left\{x \in X: S^{-1} x \neq T^{-1} x\right\}$. Let $\mathcal{M}_{1}(X)$ denote the set of all Borel probability measures on $X$.

Definition 1. The uniform topology $\tau$ is defined by the base of neighborhoods $\mathcal{U}=\left\{U\left(T ; \mu_{1}, \mu_{2}, \ldots, \mu_{n}\right)\right\}$ where

$$
U\left(T, \mu_{1}, \mu_{2}, \ldots, \mu_{n}, \varepsilon\right)=\left\{S \in \operatorname{Homeo}(X) \mid \mu_{i}(E(S, T))<\varepsilon, i=1, \ldots, n\right\},
$$

$T \in \operatorname{Homeo}(X), \mu_{1}, \mu_{2}, \ldots, \mu_{n} \in \mathcal{M}_{1}(X)$, and $\varepsilon>0$.

Homeo $(X)$ is a Hausdorff topological group with respect to $\tau$.

Remark that if one considers the set $E_{0}(T, S)=\{x \in X: S x \neq T x\}$ instead of $E(T, S)$ in the definition of $\tau$, then the topology defined by $E_{0}(T, S)$ is equivalent to $\tau$.

The main result of this paper is the Rokhlin lemma proved for an arbitrary aperiodic homeomorphism of Cantor dynamics. Let $\mathcal{A} p$ denote the set of all aperiodic homeomorphisms of $X$.

Theorem 2. Let $T \in \mathcal{A} p, \mu_{1}, \ldots, \mu_{k} \in \mathcal{M}_{1}(X), \varepsilon>0$, and $n \geq 2$. Then there exists a clopen set $E \subset X$ such that sets $E, T E, \ldots, T^{n-1} E$ are pairwise disjoint and

$$
\mu_{i}\left(E \cup T E \cup \ldots \cup T^{n-1} E\right)>1-\varepsilon \quad \text { for all } 1 \leq i \leq k .
$$

In contrast to measurable dynamics, we do not assume that $\mu_{i}$ is invariant (or non-singular) with respect to $T$. Furthermore, we prove in Theorem 2 that the base $E$ of the tower $\left(E, T E, \ldots, T^{n-1} E\right)$ can be chosen clopen.

The proof of Theorem 2 is based on the following result. It turns out that a Cantor set $X$ can be represented as a disjoint union of clopen $T$-towers of sufficiently large height where $T$ is an aperiodic homeomorphism. Then we can reconstruct these towers to get new ones such that the union of bases and tops is of small measure.

We prove several statements which are immediate consequences of Theorem 2, In particular, we will show that every aperiodic homeomorphism may be approximated in $\tau$ by periodic homeomorphisms with finite periods. We will further prove that, given $S \in \mathcal{A} p$, the set $\left\{T S T^{-1}: T \in \operatorname{Homeo}(X)\right\}$ is $\tau$-dense in $\mathcal{A} p$. This property, called the Rokhlin property, was also studied in [GK, GW, R.

\section{T-Towers COVERING a CANTOR SET}

Let $T \in \operatorname{Homeo}(X)$ and let $E$ be a clopen set such that $E \cap T^{i}(E)=\emptyset, i=$ $1, \ldots, n-1$. Then $\xi=\left(E, T E, \ldots, T^{n-1} E\right)$ is called a $T$-tower with base $E$ and top $T^{n-1}(E)$. The number $h(\xi)=n$ is the height of $\xi$, and the clopen set $C=\bigcup_{i=0}^{n-1} T^{i} E$ is called the support of $\xi$.

If $T$ is a minimal homeomorphism of a Cantor set $X$, then for every clopen set $E$ one can easily find a finite collection of disjoint $T$-towers $\Xi=\left(\xi_{1}, \ldots, \xi_{m}\right)$ such that their supports $C_{i}, i=1, \ldots, m$, cover $X$ and $E$ is the union of bases of these towers. Note that $T$ maps the union of tops of $\xi_{i}$ onto $E$.

In this section we show that a similar cover exists for any aperiodic homeomorphism $T$ of $X$. The following proposition will be used in our proof of the Rokhlin lemma.

Proposition 3. Let $T$ be an aperiodic homeomorphism of a Cantor set X. Given a positive integer $n \geq 2$, there exists a partition of $X$ into a finite number of $T$-towers $\Xi=\left\{\xi_{1}, \ldots, \xi_{m}\right\}$ such that the height $h\left(\xi_{i}\right)$ of every tower is at least $n$. 
Proof. Since $T$ is aperiodic, every point $x \in X$ has a clopen neighborhood $V_{x}$ such that the sets $V_{x}, T V_{x}, \ldots, T^{n-1} V_{x}$ are pairwise disjoint. The open cover $\left\{V_{x}: x \in\right.$ $X\}$ of $X$ contains a finite subcover $\left\{V_{1}, V_{2}, \ldots, V_{k}\right\}$. Setting

$$
\begin{aligned}
& U_{1}=V_{1}, \\
& U_{2}=V_{2}-U_{1}, \\
& \ldots \ldots \ldots \ldots \\
& U_{k}=V_{k}-\left(U_{1} \cup U_{2} \cup \ldots \cup U_{k-1}\right)
\end{aligned}
$$

we get a disjoint finite cover of $X$ by clopen sets such that $U_{i} \cap T^{j} U_{i}=\emptyset, j=$ $1, \ldots, n-1, i=1, \ldots, k$. Throughout the proof we will deal with clopen sets only.

(a) The set $\Xi$ will be constructed inductively. At the first step, we start with the $T$-tower $\xi_{1}=\left(U_{1}, T U_{1}, \ldots, T^{n-1} U_{1}\right)$.

Let $C_{1}=\bigcup_{s=0}^{n-1} T^{s} U_{1}$ be the support of $\xi_{1}$. If $C_{1}=X$, we are done. If $C_{1}$ is a proper subset of $X$, consider the sets $U_{i}^{1}=U_{i}-C_{1}, i=2,3, \ldots, k$. Note that some of the sets $U_{i}^{1}$ may be empty. Without loss of generality assume that $U_{2}^{1} \neq \emptyset$. Note that the first set from $C_{1}$ which could meet the $T$-orbit of $U_{2}^{1}$ is $U_{1}$. Define

$$
U_{2}^{1}(i)=\left\{x \in U_{2}^{1}: T^{i} x \in U_{1}, T^{j} x \notin U_{1}, 0 \leq j \leq i-1\right\}, i=1, \ldots, n-1,
$$

and

$$
U_{2}^{1}(0)=\left\{x \in U_{2}^{1}: T^{j} x \notin U_{1}, \text { for all } 1 \leq j \leq n-1\right\} .
$$

In particular, some of the sets $U_{2}^{1}(i), i=1, \ldots, n-1$, may be empty. Each set $U_{2}^{1}(i)$ is the base of the $T$-tower

$$
\xi_{2}^{1}(i)=\left\{U_{2}^{1}(i), T U_{2}^{1}(i), \ldots, T^{n-1+i} U_{2}^{1}(i)\right\}, \text { for all } i=0,1, \ldots, n-1 .
$$

We see that the first $i-1$ sets of the tower $\xi_{2}^{1}(i)$ do not intersect $\xi_{1}$ and the other sets form a subtower in $\xi_{1}$. Remark that the towers $\xi_{2}^{1}(i)$ and $\xi_{2}^{1}(j)$ are obviously disjoint whenever $i \neq j$ but each tower $\xi_{2}^{1}(i), 1 \leq i \leq n-1$, meets $\xi_{1}$. To make all of them disjoint, we delete all sets $T^{i} U_{2}^{1}(i), 1 \leq i \leq n-1$, from the base of the tower $\xi_{1}$. Take the set $U_{1}^{1}=U_{1}-\bigcup_{i=1}^{n-1} T^{i} U_{2}^{1}(i)$ as the base of a subtower of $\xi_{1}$. Denote this subtower by $\xi_{1}^{1}$. At the end of the first step, we get the collection of disjoint $T$-towers $\Xi(1)=\left\{\xi_{1}^{1}, \xi_{2}^{1}(0), \xi_{2}^{1}(1), \ldots, \xi_{2}^{1}(n-1)\right\}$, each of which has height at least $n$. Denote by $C_{1}^{1}, C_{2}^{1}(0), C_{2}^{1}(1), \ldots, C_{2}^{1}(n-1)$ the supports of corresponding towers from $\Xi(1)$.

(b) We note that $C_{1}^{1} \cup C_{2}^{1}(0) \cup C_{2}^{1}(1) \cup \cdots \cup C_{2}^{1}(n-1)=C_{1} \cup C_{2}^{1}$ where $C_{2}^{1}=$ $\bigcup^{n-1} T^{i} U_{2}^{1}$. If $C_{1} \cup C_{2}^{1}$ covers $X$, the proof is complete. If not, define the sets $\stackrel{s=0}{U_{i}^{2}}=U_{i}^{1}-C_{2}^{1}$, for all $i=3,4, \ldots, k$. It is easily seen that $\left\{C_{1}^{1}, C_{2}^{1}(0), C_{2}^{1}(1), \ldots\right.$, $\left.C_{2}^{1}(n-1), U_{3}^{2}, \ldots, U_{k}^{2}\right\}$ is a partition of $X$. Without loss of generality we assume that $U_{3}^{2} \neq \emptyset$. For each tower $\xi$ of $\Xi(1)$, put

$$
U_{3}^{2}(\xi)=\left\{x \in U_{3}^{2}: T^{i} x \text { meets } \xi \text { for some } 1 \leq i \leq n-1\right\} .
$$

Also, let $U_{3}^{2}(0)=U_{3}^{2}-\bigcup_{\xi \in \Xi(1)} U_{3}^{2}(\xi)$. Put $\eta=\left\{U_{3}^{2}(0), \ldots, T^{n-1} U_{3}^{2}(0)\right\}$. Note that $\eta$ does not intersect any $\xi \in \Xi(1)$. We can now apply the construction used in case (a) to the set $U_{3}^{2}(\xi)$ and the tower $\xi \in \Xi(1)$. This means that we can partition $U_{3}^{2}(\xi)$ into the sets $\left\{U_{3}^{2}(\xi, i): 1 \leq i \leq n-1\right\}$, where $U_{3}^{2}(\xi, i)$ contains all points whose orbits meet $\xi$ precisely at the $i$-th step. Thus, we have defined a $T$-tower $\xi(i), i=1, \ldots, n-1$, with base $U_{3}^{2}(\xi, i)$. The height of this tower is $h(\xi)+i$ where $h(\xi)$ is the height of $\xi$. The towers $\xi(i)$ remove at most $n-1$ subtowers from $\xi$. 
Denote by $\xi^{\prime}$ the remaining part of $\xi$. Thus, $\xi$ can be represented as a disjoint union of at most $n T$-towers. At the end of this step, the set $\Xi(2)$ consists of all $\xi^{\prime}, \xi(i)$ and $\eta$, where $1 \leq i<n$ and $\xi$ runs over $\Xi(1)$. Clearly, we have constructed a disjoint set of $T$-towers of heights at least $n$. The union of supports of these towers equals $C_{1} \cup C_{2}^{1} \cup C_{3}^{2}$ where $C_{3}^{2}=\bigcup_{i=0}^{n-1} T^{i} U_{3}^{2}$.

(c) If $C_{1} \cup C_{2}^{1} \cup C_{3}^{2}=X$, the proof is finished. If $C_{1} \cup C_{2}^{1} \cup C_{3}^{2}$ is a proper subset of $X$ we consider the sets $U_{j}^{3}=U_{j}^{2}-C_{3}^{2}$, for all $j=4,5, \ldots, k$, and apply the above construction again. It is obvious that after at most $k-1$ steps we will have constructed a collection of towers $\Xi$ satisfying the conditions of the proposition.

Corollary 4. Let $T$ be an aperiodic homeomorphism of X. Suppose that there exist a clopen set $F$ and $N \in \mathbb{N}$ such that $X=\bigcup_{i=0}^{N} T^{i} F$. Then for any integer $n \geq N$ there exists a finite partition $\Xi=\left\{\xi_{0}, \ldots, \xi_{m}\right\}$ of $X$ into $T$-towers of height at least $n$ such that the union of the bases of these towers is a subset of $F$.

Proof. This result can be proved by the method used in Proposition 3 To do this, we start with a finite cover $\left\{U_{1}, \ldots, U_{k}\right\}$ of $F$ by disjoint clopen sets such that $U_{i} \cap T^{j} U_{i}=\emptyset, 0<j<n, 1 \leq i \leq k$. Then we can construct disjoint clopen $T$-towers of height at least $n$ with bases containing in the $U_{i}$ 's as above. Since $X=\bigcup_{i=0}^{N} T^{i} F$ and $n \geq N$ we prove that the towers we have constructed cover $X$.

\section{Proof of the Rokhlin lemma}

This proof will be based on the decomposition of $X$ into a finite set of towers found in Proposition 3. In this proof, we again work with clopen sets only.

Proof of Theorem 2. Choose $m \in \mathbb{N}$ such that $1 / m<\varepsilon$. Using Proposition [3 find a finite set of disjoint $T$-towers $\Lambda=\left(\lambda_{1}, \ldots, \lambda_{q}\right)$, consisting of clopen sets, such that $h\left(\lambda_{i}\right) \geq n m^{k}, i=1, \ldots, q$. Denote by $V_{1}, \ldots, V_{q}$ and $B_{1}, \ldots, B_{q}$ the tops and bases of towers $\lambda_{1}, \ldots, \lambda_{q}$, respectively. It follows from the construction of towers that

$$
T\left(\bigcup_{i=1}^{q} V_{i}\right)=\bigcup_{i=1}^{q} B_{i}
$$

Fix the measure $\mu_{1}$. Let

$$
F_{l}=\bigcup_{s=1}^{q} \bigcup_{j=(l-1) n m^{k-1}}^{l n m^{k-1}-1} T^{-j} V_{s}, \quad l=1, \ldots, m .
$$

Then $F_{1}, F_{2}, \ldots, F_{m}$ are disjoint clopen sets. Amongst these sets there exists at least one of them, say $F_{l_{1}}$, whose $\mu_{1}$-measure is not greater than $1 / m$. Denote this set by $F^{l_{1}}$. By construction, $F^{l_{1}}$ consists of $q$ towers

$$
\lambda_{s}^{l_{1}}=\left\{T^{-j} V_{s}:\left(l_{1}-1\right) n m^{k-1} \leq j \leq l_{1} n m^{k-1}-1\right\}, \quad s=1,2, \ldots, q,
$$

such that $h\left(\lambda_{s}^{l_{1}}\right) \geq n m^{k-1}$. Let $V_{s}^{l_{1}}$ denote the top of the tower $\lambda_{s}^{l_{1}}, s=1,2, \ldots, q$.

Define the sets

$$
F_{l}^{l_{1}}=\bigcup_{s=1}^{q} \bigcup_{j=(l-1) n m^{k-2}}^{l n m^{k-2}-1} T^{-j} V_{s}^{l_{1}}, \quad l=1,2, \ldots, m
$$


Since $F_{1}^{l_{1}}, F_{2}^{l_{1}}, \ldots, F_{m}^{l_{1}}$ are disjoint sets, at least one of them has $\mu_{2}$-measure not greater than $1 / m$. Denote this set by $F^{l_{1}, l_{2}}$. Since $F^{l_{1}, l_{2}} \subset F^{l_{1}}$, we have that $\mu_{i}\left(F^{l_{1}, l_{2}}\right) \leq 1 / m, i=1,2$.

We continue this construction by induction. At the last step, we have found a set $F^{l_{1}, \ldots, l_{k-1}}$ which consists of $q$-towers of height at least $n m$. Denote by $V_{1}^{l_{1}, \ldots, l_{k-1}}, \ldots, V_{q}^{l_{1}, \ldots, l_{k-1}}$ the tops of these towers. Define

$$
F_{l}^{l_{1}, \ldots, l_{k-1}}=\bigcup_{s=1}^{q} \bigcup_{j=(l-1) n}^{l n-1} T^{-j} V_{s}^{l_{1}, \ldots, l_{k-1}}, \quad l=1,2, \ldots, m .
$$

As above, at least one of the sets $F_{1}^{l_{1}, \ldots, l_{k-1}}, \ldots, F_{m}^{l_{1}, \ldots, l_{k-1}}$ has $\mu_{k}$-measure not greater than $1 / \mathrm{m}$. We denote this set by $F^{l_{1}, \ldots, l_{k}}=F_{0}$. Thus, by construction of $F_{0}$, we have that

$$
\mu_{i}\left(F_{0}\right) \leq 1 / m<\varepsilon, \quad i=1,2, \ldots, k,
$$

and, moreover, $\bigcup_{j=0}^{\infty} T^{j} F_{0}=X$ (actually, this union is finite). We see that $F_{0}$ is formed by $q T$-towers which have height $n$. Let $V_{1}^{l_{1}, \ldots, l_{k}}, \ldots, V_{q}^{l_{1}, \ldots, l_{k}}$ be the tops of these towers. Denote

$$
F=\bigcup_{s=1}^{q} V_{s}^{l_{1}, \ldots, l_{k}}
$$

Then the above inequality for $\mu_{i}\left(F_{0}\right)$ implies that $\mu_{i}\left(\bigcup_{j=0}^{n-1} T^{-j} F\right)<\varepsilon$, for all $i=$ $1,2, \ldots, k$. Note that there exists $K \in \mathbb{N}$ such that $T^{K}\left(V_{s}^{l_{1}, \ldots, l_{k}}\right)=V_{s}$ for all $s=1, \ldots, q$.

Now we define a new finite collection $\Xi$ of $T$-towers whose bases are clopen subsets of $F$. Let $D_{s p}=T^{-1} B_{p} \cap V_{s}$ and $C_{s p}=T^{-K} D_{s p}, 1 \leq s, p \leq q$ (some of these sets may be empty). Clearly, $\bigcup_{s, p=1}^{q} C_{s p}=F$. Then $\left(C_{s p}, T C_{s p}, \ldots, T^{h(s p)-1} C_{s p}\right)$ constitutes a $T$-tower $\xi(s p) \in \Xi$ where the height $h(s p)$ of $\xi(s p)$ is defined from the condition $T^{h(s p)-1} C_{s p} \subset T^{-1} F, 1 \leq s, p \leq q$. We get that the set $\Xi=(\xi(s p): 1 \leq$ $s, p \leq q)$ consists of $T$-towers whose supports form a partition of $X$ and

$$
\mu_{i}\left(\bigcup_{l=0}^{n-1} \bigcup_{s, p=1}^{q} T^{h(s p)-1-l} C_{s p}\right)<\varepsilon, \quad i=1,2, \ldots, k .
$$

To finish the proof, define the clopen set $E$ :

$$
E=\bigcup_{s, p=1}^{q} \bigcup_{j=0}^{L(s p)} T^{j n} C_{s p}
$$

where $L(s p)=\left[n^{-1}(h(s p)-1)\right]-1$. It is easily seen that $E \cap T^{j} E=\emptyset, j=1, \ldots, n-1$, and

$$
\mu_{i}\left(E \cup T E \cup \ldots \cup T^{n-1} E\right)>1-\varepsilon, \quad 1 \leq i \leq k .
$$




\section{Corollaries}

We give several immediate consequences of the result proved above. The next two corollaries can be easily deduced from the proof of Theorem 2

Corollary 5. Suppose $T$ is an aperiodic homeomorphism of $X$. Let $\mu_{1}, \ldots, \mu_{k}$ be Borel probability measures, let $n \geq 2$ be a positive integer, and let $\varepsilon>0$. Then there exists a clopen partition of $X$ into a finite number of $T$-towers $\Xi=\left\{\xi_{1}, \ldots, \xi_{q}\right\}$ such that:

(i) for every tower $\xi \in \Xi, h(\xi) \geq n$;

(ii) for $i=1, \ldots, k$,

$$
\mu_{i}\left(\bigcup_{s=1}^{q} \bigcup_{j=0}^{h\left(\xi_{s}\right)-n} T^{j} B_{s}\right)>1-\varepsilon
$$

where $B_{s}$ is the base of $\xi_{s}$.

Corollary 6. Suppose $T$ is an aperiodic homeomorphism of $X$. Let $\mu_{1}, \ldots, \mu_{k}$ be Borel probability measures and let $\varepsilon>0$. Then there exists a clopen set $A$ such that $\bigcup_{j \geq 0} T^{j} A=X$ and $\mu_{i}(A)<\varepsilon, i=1, \ldots, k$.

Let $T$ be an aperiodic homeomorphism of a Cantor set $X$. We recall the definition of the full group $[T]$ of homeomorphisms generated by $T$. By definition, a homeomorphism $\gamma \in \operatorname{Homeo}(X)$ belongs to $[T]$ if the sets $X_{n}=\left\{x \in X: \gamma x=T^{n} x\right\}$ form a partition of $X$. In other words, there exists an integer-valued function $n(x)$ such that $\gamma x=T^{n(x)} x$ for all $x \in X$.

Let $\mathcal{P e r}$ denote the set of pointwise periodic homeomorphisms of $\mathrm{Homeo}(X)$. Consider the subset $\mathcal{P e r}_{0} \subset \mathcal{P}$ er consisting of all homeomorphisms with finite period, that is $P \in \mathcal{P} e r_{0}$ if and only if there exists $m \in \mathbb{N}$ such that $P^{m} x=x$ for all $x \in X$. This means that $X$ can be decomposed into a finite union of sets $X_{p}$ such that the period of $P$ at each point from $X_{p}$ is exactly $p$.

The following statement shows that every aperiodic homeomorphism can be approximated by a periodic homeomorphism in the uniform topology. This result has analogues in ergodic theory and Borel dynamics.

Corollary 7. Let $T$ be an aperiodic homeomorphism and let

$$
U(T)=U\left(T ; \mu_{1}, \ldots, \mu_{n} ; \varepsilon\right)
$$

be a $\tau$-neighborhood of $T$. Then there exists a periodic homeomorphism $P \in \mathcal{P e r}_{0}$ such that $P \in U(T) \cap[T]$.

Proof. Apply Corollary 5 for $n=2$. We obtain a clopen partition of $X$ into a finite number of towers $\Xi=\left\{\xi_{1}, \ldots, \xi_{q}\right\}$ such that for all $i=1, \ldots, k$

$$
\mu_{i}\left(\bigcup_{j=1}^{q} \bigcup_{l=0}^{h_{j}-2} T^{l} B_{j}\right)>1-\varepsilon,
$$

where $B_{j}$ and $h_{j}$ are the base and the height of $\xi_{j}$, respectively. Now we can define the periodic homeomorphism $P$ as follows:

$$
P x= \begin{cases}T x, & \text { if } x \in \bigcup_{j=1}^{q} \bigcup_{l=0}^{h_{j}-2} T^{l} B_{j}, \\ T^{-\left(h_{j}-1\right)} x, & \text { if } x \in T^{h_{j}-1} B_{j} \text { for some } j=1, \ldots, q .\end{cases}
$$


Obviously, $P \in[T] \cap \mathcal{P} e r_{0}$. It is easy to see that $P$ also belongs to $U(T)$.

Corollary 8. $\mathcal{A p}$ is a closed nowhere dense subset in $(\operatorname{Homeo}(X), \tau)$.

Proof. It was proved in BDK2 that $\mathcal{A} p$ is closed in $(H o m e o(X), \tau)$. It follows from Corollary $\square$ that $\mathcal{A} p$ is a subset of $(\operatorname{Homeo}(X), \tau)$ with empty interior.

Corollary 9. Let $S \in \mathcal{A} p$. Then, for any $R \in \mathcal{A} p$ and $U(R)=U\left(R ; \mu_{1}, \ldots, \mu_{p} ; \varepsilon\right)$, there exists some $T \in H$ omeo $(X)$ such that $T^{-1} S T \in U(R)$. In other words, Ap is the $\tau$-closure of $\left\{T^{-1} S T: T \in \operatorname{Homeo}(X)\right\}$.

Proof. Given $R \in \mathcal{A} p, \mu_{1}, \ldots, \mu_{p} \in \mathcal{M}_{1}(X)$, and $\varepsilon>0$, apply Corollary 5 with $n=$ 3. We get a clopen partition of $X$ into a finite number of $R$-towers $\Xi=\left\{\xi_{1}, \ldots, \xi_{q}\right\}$ such that

$$
\mu_{i}\left(\bigcup_{j=1}^{q} \bigcup_{k=0}^{h_{j}-3} R^{k} B_{j}\right)>1-\epsilon, \quad i=1,2, \ldots, q,
$$

where $B_{j}$ is the base of $\xi_{j}$ and $h_{j}$ is its height.

Put $M=\max \left\{h_{j}: 1 \leq j \leq q\right\}$. Apply Proposition 3 with $n=M$ to the homeomorphism $S \in \mathcal{A} p$ and find a clopen partition of $X$ into a finite number of $S$-towers $\Lambda=\left\{\lambda_{1}, \ldots, \lambda_{l}\right\}$ such that the height $n_{i}$ of each tower $\lambda_{i}$ is at least $M$. We can refine either $\Xi$ or $\Lambda$ to get the same number of towers in $\Xi$ and $\Lambda$. Thus, without loss of generality, we can set $q=l$. Denote by $Z_{j}$ the base of $\lambda_{j}$. Let $Q_{j}$ be a homeomorphism which maps $B_{j}$ onto $Z_{j}, j=1, \ldots, q$, and let $Q$ be a homeomorphism which sends $\bigcup_{j=1}^{q} R^{h_{j}-1} B_{j}$ onto $\bigcup_{j=1}^{q} \bigcup_{k=h_{j}-1}^{n_{j}-1} S^{k} Z_{j}$. Define the homeomorphism $T$ as follows:

$$
T x= \begin{cases}S^{i} Q_{j} R^{-i}, & \text { if } x \in R^{i} B_{j}, 0 \leq i \leq h_{j}-2,1 \leq j \leq q, \\ Q x, & \text { if } x \in \bigcup_{j=1}^{q} R^{h_{j}-1} B_{j} .\end{cases}
$$

In such a way, the homeomorphism $T$ is defined on the set $X$. It is not hard to see that $E_{0}\left(R, T^{-1} S T\right)=\left\{x \in X: R x \neq T^{-1} S T x\right\} \subset \bigcup_{j=1}^{q} \bigcup_{i=1,2} R^{h_{j}-i} B_{j}$. Therefore, $T^{-1} S T \in U(R)$.

\section{ACKNOWLEDGEMENT}

We thank J. Feldman, A. Kechris, J. Kwiatkowski, and B. Miller for helpful discussions. The first-named author thanks the University of New South Wales for the warm hospitality and the Australian Research Council for its support.

\section{REFERENCES}

[AHK] E. Akin, M. Hurley, J. Kennedy, Dynamics of topologically generic homeomorphisms, Memoirs of the AMS, 164, (2003). MF1980335 (2004j:37024)

[AP] S. Alpern, V.S. Prasad, Typical Dynamics of Volume Preserving Homeomorphisms, Cambridge University Press, 2000. MR 1826331 (2002i:37006)

[BDK1] S. Bezuglyi, A.H. Dooley, J. Kwiatkowski, Topologies on the group of Borel automorphisms of a standard Borel space, preprint, 2003.

[BDK2] S. Bezuglyi, A.H. Dooley, J. Kwiatkowski, Topologies on the group of homeomorphisms of a Cantor set, preprint, 2003. 
[BK1] S. Bezuglyi, J. Kwiatkowski, The topological full group of a Cantor minimal system is dense in the full group, Topological Methods in Nonlinear Analysis, 16 (2000), 371 - 397. MR.1820514 (2002b:37012)

[BK2] S. Bezuglyi, J. Kwiatkowski, Topologies on full groups and normalizers of Cantor minimal systems, Math. Physics, Analysis, and Geometry, 9 (2002), No. 3, 1 - 10. MR 1949801 (2003j:37014)

[FL] J. Feldman, D. Lind, Hyperfiniteness and the Halmos-Rohlin theorem for nonsingular abelian actions, Proc. Amer. Math. Soc., 55 (1976), 339 - 344. MR0409764 (53:13516)

[EP] S.J. Eigen, V.S. Prasad, Multiple Rokhlin tower theorem: a simple proof, New York J. Math., 3A (1997(98)), 11 - 14. MR.1604573(99h:28032)

[GK] E. Glasner, J. King, A zero-one law for dynamical properties, Topological Dynamics and Applications, Contemp. Math., 215 (1998), 215 - 242. MR603201 (99d:28039)

[GW] E. Glasner, B. Weiss, The topological Rohlin property and topological entropy, Amer. J. Math., 123 (2001), 1055 - 1070. MR1867311 (2002h:37025)

[LW] E. Lehrer, B. Weiss, An $\varepsilon$-free Rokhlin lemma, Erg. Th. Dyn. Syst., 2 (1982), 45 - 48. MR0684243 (84e:28021)

[OW] D. Ornstein, B. Weiss, Ergodic theory of amenable group actions: I. Rohlin lemma, Bull. Amer. Math. Soc., 2 (1980), 161 - 164. MR0551753 (80j:28031)

[R] V.A. Rokhlin, Selected topics from the metric theory of dynamical systems (Russian), Uspehi Matem. Nauk (N.S.), 4 (1949), no. 2, 57-128. MF030710(11:40b)

Institute for Low Temperature Physics, National Academy of Sciences of Ukraine, KHARKOV, UKRAINE

E-mail address: bezuglyi@ilt.kharkov.ua

School of Mathematics, University of New South Wales, Sydney, Australia

E-mail address: a.dooley@unsw.edu.au

Institute for Low Temperature Physics, National Academy of Sciences of Ukraine, Kharkov, Ukraine

E-mail address: medynets@ilt.kharkov.ua 